\title{
SONIFICATION, MUSIFICATION, AND SYNTHESIS OF ABSOLUTE PROGRAM MUSIC
}

\author{
Allan D. Coop \\ P.O. Box 5160, Braddon, 2612, Australia \\ allan.coop@gmail.com
}

\begin{abstract}
When understood as a communication system, a musical work can be interpreted as data existing within three domains. In this interpretation an absolute domain is interposed as a communication channel between two programatic domains that act respectively as source and receiver. As a source, a programatic domain creates, evolves, organizes, and represents a musical work. When acting as a receiver it re-constitutes acoustic signals into unique auditory experience. The absolute domain transmits physical vibrations ranging from the stochastic structures of noise to the periodic waveforms of organized sound. Analysis of acoustic signals suggest recognition as a musical work requires signal periodicity to exceed some minimum. A methodological framework that satisfies recent definitions of sonification is outlined. This framework is proposed to extend to musification through incorporation of data features that represent more traditional elements of a musical work such as melody, harmony, and rhythm.
\end{abstract}

\section{INTRODUCTION}

A conceptual framework is proposed for the organization and description of relationships between a musical work, digital data, and sound. The framework encompasses and organises digital data and acoustic signals that range from noise to musical sound. The goal is to employ this framework to develop a methodology suited to guide the creation and evolution of acoustic signals through sonification to achieve musification.

To this end, acoustic signals, can be better understood by, (i) modelling them as components of a communication system, and (ii) quantitatively locating such signals within the compass of their stochastic and periodic components. It is hypothesised that this is best achieved by employing both basic measures of communication theory such as the efficiency or redundancy of signal components and statistical features of the autocorrelation function of an acoustic signal. In doing so, a methodology is identified whereby any digital content or data stream can be evolved into acoustically organized musical experiences.

Music has a long and complicated history of development in the analog domain. However, its relationship to, development, and analysis within the digital domain is now the focus of intense study. This digital domain is a result of phenomenal advances in computer and communications technology made possible by the continued elaboration of integrated circuit processing technology.

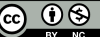

This work is licensed under Creative Commons Attribution Non Commercial 4.0 International License. The full terms of the License are available at http://creativecommons.org/licenses/by-nc/4.0
It is a technology that has increasingly replicated the functionality of traditional analog devices, including the digital replication of acoustic signal sources such as, for example, those of musical instruments. The impact of communication theory on these developments has been immense [1].

Complex computer-based implementations of digital hardware provide many advantages; programmability, flexibility, additional product functionality such as analog to digital conversion (and vice versa), short design cycles, and good immunity to both noise and manufacturing process tolerances [2].

Such technological change has propelled development and research in sonification [3, 4] and musification [5, 6, 7]. Sonification was originally described as, the use of synthetic non-verbal audio to support information processing activities [8]. Subsequently, sonification has been employed to, (i) transform the inaudible into the audible, (ii) employ audition to gain knowledge, and (iii) develop listening techniques for scientific inquiry [9]. It has more recently been defined as a technique that uses data as input and generates sound signals, with the caveats that, (i) the sound reflects objective properties or relations in input data, (ii) the transformation is systematic, (iii) the sonification is reproducible, and (iv) the system can intentionally be used with different data [10].

Musification has been defined as the musical representation of data [7]. It is designed to go beyond direct sonification and include elements of tonality and the use of modal scales to create musical auralizations. The resulting musical structures take advantage of higher-level musical features such as polyphony and tonal modulation in order to engage the listener [11].

More recently it has been proposed that full realization of the potential of sonification must also include, "the craft and art of music composition" [5]. It is in this sense that here threads of this "second order of intervention" [5] are explored. The aim is to, (1) introduce elements of a compositional framework and methodology for the evolution of sonification into musification as a means of artistic expression and (2) better understand powerful, poorly defined, and still under-examined aspects of organized sound.

\section{THEORY}

Two propositions inform the framework developed here. (1) Messages within a communication system are transmitted through one or more channels connecting a source to a receiver [1]. (2) A received message exhibits two aspects, one is semantic (having a universal logic, structured, articulable, translatable, and serving the behaviorist conception of action preparation), the other esthetic (untranslatable as there is no language available to translate it to, it refers to the repertoire of knowledge associated with the particular communication system) [12].

The framework is then evolved from the proposal that two axiomatic and mutually exclusive domains together form the organi- 
zational foundation of a musical work (c.f. [13]). One domain is internal, cognitively located, subjectively individual, and referred to as programatic. The other is external, purely physical, collectively objective, quantifiable, and referred to as absolute. This latter domain essentially involves nothing but a section of the theory of the motions of elastic bodies [14].

The internal programatic domain is physiological and psychological. It comprises the totality of the abstract, conceptual aspects of a musical work, including its intellectual creation and non-acoustic or symbolic representations, whether memorised or physically recorded. Within this domain are found the responsible originating creative processes which are employed to generate, organise, produce, and subsequently interpret and re-fabricate acoustic signals into a musical work. Due to the extreme scale and complexity, thus difficulty, of investigating the processes involved, little is currently understood about the details by which these functions and behaviors are realized (see, for example [15]).

The programatic domain extends until physical vibration is initiated in the external world. It is this acoustic signal which forms the absolute component of a musical work. It originates as the programmatic component is transferred into the absolute domain. The resultant waveform exists as a temporal sequence of physical vibrations in the world. It ranges from one that: (a) Can be resolved via Fourier's theorem [16] into one or more sine waves each exhibiting a single frequency, to an acoustic signal that is essentially noise as it, (b) exhibits stochastic repetition in the absence of correlation of amplitude or interval of succession [17]. In contrast with the programatic domain, much detail is known about the physical aspects of the absolute domain (see, for example [18]).

In other words, as a musical sound an acoustic signal is primarily comprised of a structured complex of periodic waveforms that exhibit a non-zero autocorrelation function. It is in this general sense that music may be thought of as organised sound (as proposed by [19]). Alternatively, as noise, an acoustic signal is primarily stochastic and exhibits an autocorrelation function which approaches zero.

Described in this way, it is clear that absolute and programatic components may be combined to form the complex acoustic signal instantiantable within individual musical works. They originate and exist within two individual and mutually exclusive domains, each required for the complete existence, communication, and experience of a musical work.

Such an interpretation allows a musical work to be characterised as a communication system (figure 1). In this view, a musical work is revealed as a triadic sequence. This sequence is, (A) initiated as a cognitive creation which is then evolved and elaborated as a programatic component within the programatic domain. At some moment, this component is, (B) physically instantiated and transmitted acoustically as the absolute component of a musical work within the absolute domain. This transmission may subsequently be received, (C) to cognitively be reconstructed as a programatic component within the programatic domain with the potential to animate the experience and behavior of a listener.

Within this communication system, an information source, here the cognitively generated programatic component of a musical work, is operated on by a transmitter; for example, human cognitive neural activity may be converted into motor activity to generate and transmit an acoustic signal either vocally or with the aid of an instrument. This signal physically propagates as the absolute component of a musical work to any receiver or human listener. Successful reception of the acoustic signal leads to the

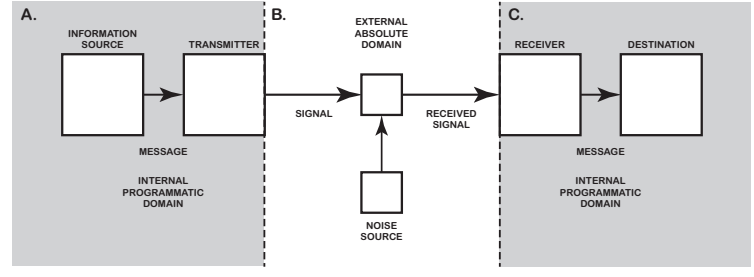

Figure 1: Relationship between the components of a musical work. A: The internally located programatic domain is the cognitive source of the programatic component of a musical work. This component is instantiated and released as an acoustic signal into the world by the transmitter. B: The acoustic signal comprises the absolute component of a musical work and propagates externally in the world until it either dissipates or is detected by a receiver. C. At the receiver, the absolute component is captured and reconstructed along with any noise to form a novel programatic component prior to reaching the cognitively located destination of conscious perception. Note: A and C may be the same individual or one or more nonbiological devices.

inverse operation done by the transmitter being performed on the signal whereby the absolute component is cognitively re-fabricated as a programatic component to exist and be experienced as a new version of the original pretransmission programatic component.

It is further proposed that this communication system has increasingly come to be located between two mutually exclusive physical domains. (i) A continuous analog domain embedding the objects of the physical world, including the human brain (or programatic domain), and more recently, (ii) A discrete mechanical or digital domain which originated with the development of communication technology.

The digital domain relies on the mathematical formalism of communication theory [1]. This theory allows both analog and digital signals to be characterized by measures of entropy and redundancy. Accordingly, a stochastic signal exhibits the highest possible entropy and thus the lowest redundancy. When digitized, such a signal typically exhibits high variability in the record of its sampled waveform. Alternatively, a periodic signal exhibits lower entropy, thus higher redundancy, and the sampled waveform exhibits lower variability.

Autocorrelation is a mathematical tool that can be employed to find and quantify the extent of repeating patterns, particularly the periodicity of a signal. It provides a measure of the similarity between observations or digital samples as a function of the lag between them. A quantitative estimate of signal periodicity (the autocorrelation coefficient) can be obtained from the autocorrelation function (the normalized autocovariance function [20]).

\section{SONIFICATION}

In the case of sonification, it is possible to base an organisational framework for a musical work on reinterpretation of pre-existing musically related ideas. Thus, for example, when considered as a song or an ode, music has been claimed to consist of three partsthe words, the melody, and the rhythm [21]. However, here the claim is that the only component suited for direct translation by sonification is that of the symbols of a data stream. Although, melodic and rhythmic components might be extracted from a data stream; for the purposes of sonification, it is hypothesised they are 
properly considered more abstract second order properties, as they are not events in the same primary way that the numeric values or symbols comprising a data stream are. It is for this reason that melody and rhythm are considered likely more properly left to be treated as components of musification.

\subsection{Implementation}

The framework and methodology proposed here are claimed to satisfy the four formal requirements for sonification. For the given example these are, (i) the objective data properties of sample redundancy and pitch frequency, (ii) the systematic transformation of data sequences to redundancy and pitch frequency, (iii) the reproducibility introduced by a mathematical model, and (iv) the applicability of the methodology to alternative data sets. The primary motivation for the approach is the aparent lack of general principle suited to guide the sonification process.

While keeping the caveats with regard to periodicity and variability introduced above in mind, importantly, the method now outlined may be generalized to convert and organise any digitized content prior to evolving it to musification. Here, it is developed for application to a symbol stream such as might be found in a file containing digitized text.

The computational implementation proceeds in two steps. (1) A series of partial redundancy values are mapped to a sequence of pitch frequencies. (2) The derived pitch frequencies can then be mapped back to refabricate the original sequence of symbols as a sequence of sounds.

For this analytic approach the absolute component of a given communication system (the data) is assumed to be constrained to the scope of a clearly defined symbol set with ergodic properties.

If this is accepted, the mathematical formalism of communication theory defines the Shannon entropy $(h)$ given in bits as

$$
h=-\sum_{i=1}^{N} p_{i} \log _{2}\left(p_{i}\right),
$$

where $p_{i}$ is the probability of the $i$ th value in a data set comprised of $N$ unique values.

As entropy is an extensive quantity, a 'normalized' entropy measure is employed to allow more meaningful comparison of different data sets. This measure, the redundancy $(r)$, is calculated as

$$
r=1-\frac{h}{H},
$$

where $H$ is the maximum or Hartley entropy [22] of the acoustic signal, calculated as

$$
H=\log _{2}(N)
$$

\subsection{Letters and words}

The partial entropy $\left(h_{i}\right)$ of each symbol is obtained from [23]

$$
h_{i}=-p_{i} \log _{2}\left(p_{i}\right),
$$

while the partial redundancy $\left(r_{i}\right)$ is determined by direct substitution of $h_{i}$ for $h$ in [2].

Once calculated, the partial redundancy can be employed to order, as required, the unique symbols within a data set.

In the case of data such as text, although not required, one initial simplification is to assume that individual letters and words are uncorrelated. Here, the letters of a text source can be directly quantified from [1-3].

The probability of each of the letters forming an individual word can be employed to calculate the partial entropy of a given word from

$$
h_{m}=\prod_{j=i}^{n} p_{j} \log _{2}\left(p_{j}\right),
$$

where $j$ gives the letter number and $n$ the number of letters in the $m$ th word in a list of unique source words. After substitution, the word probabilities of the originating data set are re-normalized prior to redundancy calculations via equations [1-3].

Alternatively, word probabilities can be obtained directly from a list of source word frequencies obtained from the given data set. For a sufficiently large sample the difference in the partial entropy calculated for words, either from individual letters or for the words them-self, indicates the extent to which letters within a given word are correlated. This provides an estimate of the bias introduced if, as a simplification, it is assumed that the letters forming a word are uncorrelated.

Autocorrelation analysis employed the xcorr function in the signal package of GNU Octave version 3.8.0-1 [24]. Raw autocorrelation functions were normalized by the correlation at lag zero using the coeff flag. The central peak of each scaled autocorrelation function was removed and the amplitude of the largest remaining positive peak was converted to a percentage and reported.

\subsubsection{Defining the digital pitch sequence}

It is widely accepted that auditory perception extends from 20 $20,000 \mathrm{~Hz}$ [18], although, for younger people, the auditory range may extend from 16-25,000 Hz [25]. Humans can sense vibrations with a frequency as low as $2 \mathrm{~Hz}$, although a minimum of about $20 \mathrm{~Hz}$ is required for perception of "tonality" [26]. Assuming the amplitude is sufficient, lower frequencies are typically felt through their vibratory effect, rather than heard.

The range of perceptually discriminable frequencies is less extensive than the range of detectable frequencies. Within the 16$16,000 \mathrm{~Hz}$ range $(16.0 \mathrm{kHz}-$ kilohertz) it has been estimated the human auditory system can distinguish about 1,200 [27] to 1,400 [28] distinct pitch levels. Increasing the range to $20 \mathrm{~Hz}-20 \mathrm{kHz}$ has little perceptual effect as only about 1,500 pitches may be discriminated [29].

For comparison with human audition, one of the largest frequency ranges of any instrument-the piano-has 88 semitones which range from $27.5 \mathrm{~Hz}-4.185 \mathrm{kHz}$, while the reproduction of orchestral music with subjectively perfect fidelity requires a frequency range of $40 \mathrm{~Hz}-15 \mathrm{kHz}$ [30].

For the purposes of developing an absolute pitch sequence for the digital domain, the human sensory capacity can be assumed to extend from silence (by definition $0 \mathrm{~Hz}$ ) to vibrations with a frequency of $16.384 \mathrm{kHz}$. For both convenience and simplicity, a lower bound of $1 \mathrm{~Hz}$ is chosen (more detail is given below). The upper bound is chosen for several reasons. It is known that human audition deteriorates with age by loss of higher frequencies. The ability to discriminate frequencies is greatly reduced above about $15 \mathrm{kHz}$, and an upper bound of $16.384 \mathrm{kHz}$ can conveniently be expressed as $2^{14}$, where the exponent immediately gives the octave count from $1 \mathrm{~Hz}$.

More technical reasons for the choice of upper bound include, (1) positive powers of 2 are important in computer science-there 


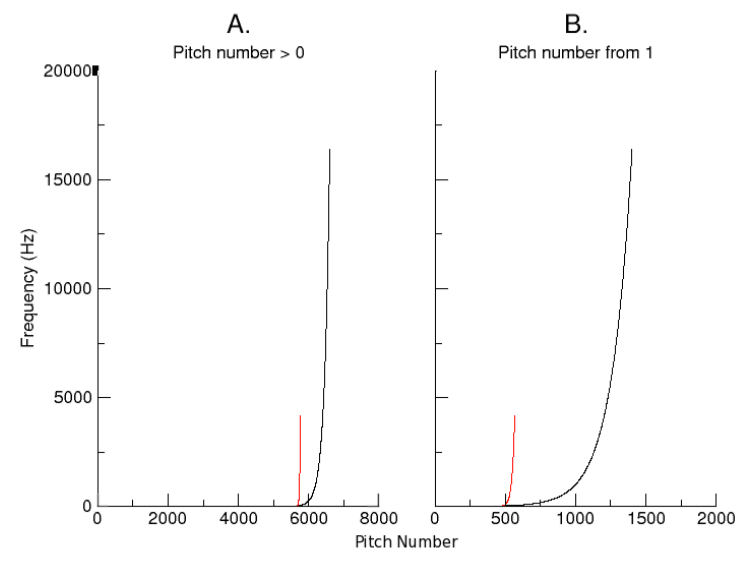

Figure 2: Relationship between absolute and unitary pitch sequences. A: Absolute (black). Frequency profile for 6,601 pitch numbers obtained when the absolute digital pitch is calculated from silence $(>0 \mathrm{~Hz})$ to $16.384 \mathrm{kHz}$. B: Unitary (black). Frequency profile for 1,401 pitch numbers when the unitary digital pitch is calculated from $\geq 1 \mathrm{~Hz}$. A, B: Classical (red). Frequency profile for each of the 88 different pitches represented by the piano keyboard. It has been transposed to match the equivalent frequency range of the associated digital pitch sequence. See text for more details.

are $2^{n}$ possible values for a $n$-bit binary register, (2) more importantly, $\log _{b}(n)$ bits are required to represent a variable that can take one of $n$ values if $n$ is a power of $b$ (when $b=2$ the units of Shannon entropy are commonly referred to as bits), (3) the logarithm of a probability distribution is useful as a measure of entropy as it is additive for independent sources, and (4) such an upper bound gives a range of 14 octaves which is similar to the 10 octaves obtained if a frequency range of $20 \mathrm{~Hz}-20.48 \mathrm{kHz}$ or $16 \mathrm{~Hz}-16.38 \mathrm{kHz}$ were to be assumed.

The foregoing considerations have led to the selection of frequencies suited for fabrication of what is referred to as a digital pitch sequence. Based on its starting frequency, this sequence may be defined as either absolute $(0 \mathrm{~Hz}-16.384 \mathrm{kHz})$ or unitary $(1 \mathrm{~Hz}-$ $16.384 \mathrm{kHz}$ ).

The relation of these two pitch sequences to the classical pitch sequence, as given by a tuned piano, is illustrated in figure 2 . This figure shows the extension of the classical range of pitch sequence to the absolute (A) and unitary (B) pitch sequences.

The absolute pitch sequence illustrated in panel (A) shows that almost $85 \%(84.9 \%)$ of frequencies are less than $16 \mathrm{~Hz}$. Alternatively, panel (B) illustrates that, for the unitary sequence, an increase in the start of the sequence from $0 \mathrm{~Hz}$ to $1 \mathrm{~Hz}$ results in more than $70 \%(71.4 \%)$ of the frequencies being greater than $16 \mathrm{~Hz}$.

\subsubsection{Mapping redundancy to frequency}

In the simplest case, once obtained, the first $m$ partial redundancy values for either words or letters can be sorted into an ordered list and mapped to the equivalent number of values obtained, as appropriate, from either the absolute or unitary frequency ranges or, more conventionally, the Classical pitch sequence of the piano keyboard. Alternatively, partial redundancy values could be mapped to either the related notes of one or more instruments or to computationally fabricated timbres.

The second and final step in the methodology is to complete the sonification process by mapping the partial redundancy $\rightarrow$ frequency relations back into the sequential order of the original data sequence.

\section{MUSIFICATION}

The methodological implementation of musification is concerned with both the absolute and programatic components of a musical work. In the development of absolute program music, the sound content is structured by reference to communication theory. This mathematical formalism is employed as a tool to assist with translation and organisation of the symbols of a digital data set into organized musical sound.

\subsection{Melody (and harmony)}

A simple melodic component is automatically generated when the symbols of a data sequence (here either letters or words) are mapped to a given pitch sequence. A least two considerations should be taken into account prior to performing this mapping. The first is that frequencies of higher pitch are more difficult to discriminate than frequencies of lower pitch. The second is that the higher the redundancy of a symbol, the less information it communicates. Thus, to transparently increase human interpretability, signals with a high rate or frequency should exhibit less variability (higher redundancy) than signals with a low rate or frequency.

As a consequence, one musification option is to map the most redundant symbols to the highest note pitches or frequencies and vice versa. Although, as for the steps given above, many other mappings might be employed.

An alternative approach is to generate a harmonic component by forming a mapping between pitch and the letters within each word. Here, the sequential partial redundancy values related to individual letters can be mapped to a given pitch sequence. The letters in each word can then provide the synchronous events that comprise individual 'chords,' i.e. a chord is given by the letters forming each successive word in a text stream.

An important step in moving from the sonification to the musification of a data stream is the addition of the relevant harmonic series to each note. Further, the acoustic properties of a given note can also be enhanced by the addition of a temporal envelope for the frequency wave forms comprising that note (see for example figure 3).

\subsection{Rhythm}

In the given example, the most direct source of rhythm is that of the poetic meter of the words in the source text. This can be characterized and applied to organise the rhythmic components of the musification process. Alternatively, where there is no obvious metric source, as might particularly be the case for non-linguistic data, the intervals between repeated symbols provide one immediate option which could be employed to organize temporal patterns. The modulation of these intervals can further be manipulated by the introduction of temporal envelopes. The effect of such a modulation is illustrated in figure 3 for a $1 \mathrm{~Hz}$ sine wave. The addition of an exponential temporal envelope reduces the effective duration of the sound from $7 \mathrm{~s}$ to about $4.5 \mathrm{~s}$. It is noted that many 


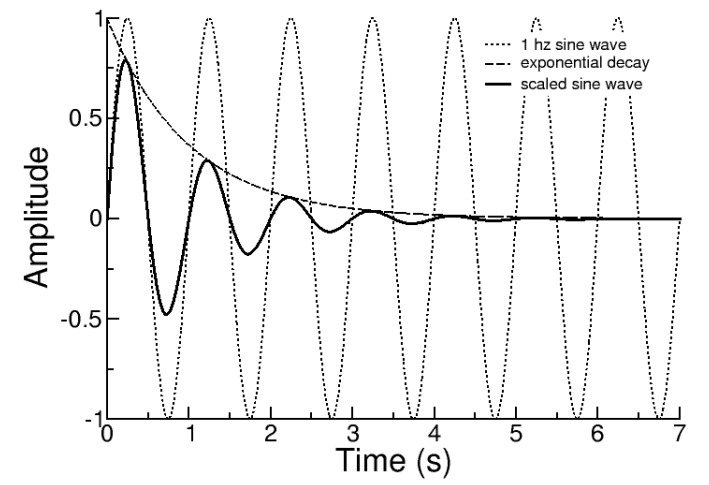

Figure 3: A simple musification technique illustrated by the effect of a temporal envelope on the magnitude and duration of a given waveform. An exponential decay (dashed line) has been applied to a sine wave (dotted line) with a frequency of $1 \mathrm{~Hz}$ and duration of $7 \mathrm{~s}$. The resultant waveform is indicated (unbroken line). The audible duration of the modulated sound is reduced from $7 \mathrm{~s}$ to about $4.5 \mathrm{~s}$ when compared with the duration of the unmodulated sine wave.

other forms of temporal envelope are available for the generation and implementation of rhythmic control beyond the duration of the raw unmodulated sound associated with sonification.

\section{RESULTS}

To characterize an acoustic signal, the redundancy and the autocorrelation coefficient of each data set, as respective measures of signal variability and signal periodicity, can be calculated and plotted.

Figure 4 illustrates such a plot for a selection of data sets that represent examples of different sounds and musical genres (see symbol $\times$ ). As might be expected for a near uniform distribution of white noise (a), the entropy is close to the Hartley entropy and consequently the redundancy is vanishingly small $(0.03 \%)$. In the absence of the zero lag peak, the autocorrelation coefficient is also everywhere near zero (largest value $0.011 \%$ at a random lag value of $47.1 \mathrm{~s}$ ).

Alternatively, a sine wave would be expected to exhibit a lower entropy than white noise due to data clustering at similar values near positive and negative peaks. Thus, a $440 \mathrm{~Hz}$ sine wave exhibits a redundancy of $34.7 \%$ while the autocorrelation coefficient is near unity $(\mathrm{g})$.

The remaining analyses of the musical works illustrated in figure 4(b)-(f) form two groups linked by (e) a gregorian chant (Kyrie Eleison). One of these groups consists of: (b) a modern electric trio (guitar, bass, and drums), (c) an aria from the Magic Flute, and (e) the gregorian chant. These musical works exhibit similar autocorrelation coefficients (respectively $23 \%, 23 \%$, $21 \%$ ) but are distinguished by their redundancies (respectively $12 \%, 14 \%, 20 \%)$. Almost orthogonal to this group is one consisting of (d), (e), and (f). These data respectively comprise one of the first works of musique concrete (Etude Aux Chemins De $\mathrm{Fer}$ ), the gregorian chant, and a minimalist work for piano and violin (Spiegel Im Spiegel). These three musical works exhibit

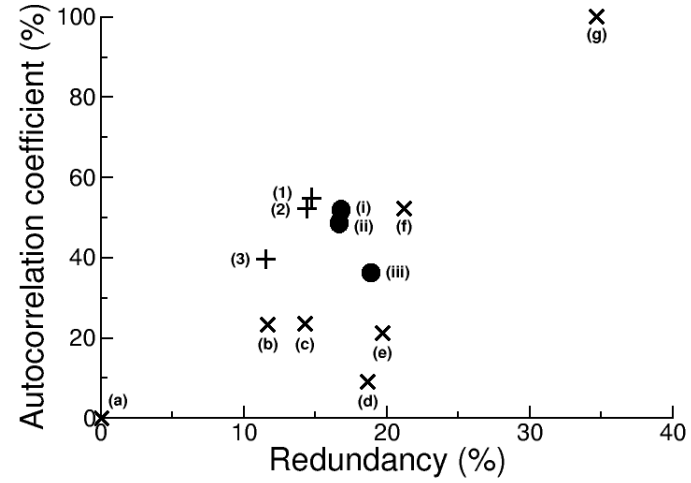

Figure 4: Relationship between the redundancy and autocorrelation coefficient of an acoustic signal. Symbol $\times$ : (a) Stochastic signal: White noise. Musical works, (b) “*****,*****”: Study For Falling Apart, (c) Mozart: The Magic Flute, Act II, Der Hölle Rache, (d) Schaeffer: Etude Aux Chemins De Fer, from Cinq Études De Bruits, (e) Gregorian Chant: Kyrie Eleison, Benedictine Monks of Santo Domingo de Silos, (f) Pärt: Spiegel Im Spiegel. (g) Periodic signal: $440 \mathrm{~Hz}$ sine wave. Sonified text (+): (1) Classical pitch sequence, (2) Unitary pitch sequence, (3) Mono pitch sequence $(1+2)$. Musified text $(-$-examples of absolute program music), (i) Unitary pitch sequence, (ii) Classical pitch sequence, (iii) Mono pitch sequence $(1+2)$. As the reported values for the autocorrelation coefficient and the redundancy lie in the range $0-1$, they are given as percentages. See text for further details.

similar redundancies (respectively 19\%, 20\%, $21 \%$ ) but are distinguished by their autocorrelation coefficients (respectively $9 \%$, $20 \%, 52 \%$ ).

For comparison with the foregoing results, figure 4 also provides results of an analysis of the sonification $(\boldsymbol{+})$ and musification (-) of a text (the song of Orpheus as reported in [31]). These data (1-3 and i-iii, respectively) exhibit similar periodicity and redundancy to the previously described musical works ( $\mathrm{a}-\mathrm{g}$ ). Musification has little effect on the periodicity data when it is sonified (1 c.f. i, 2 c.f. 2,3 c.f. iii), but increases the redundancy of the signal, particularly when the individual data sets for unitary and classical pitch sequences are combined to form a single acoustic sequence (see figure 4, 3 c.f. iii, and 1 c.f. i and 2 c.f. ii).

\section{DISCUSSION}

Initially, the idea of a musical work being an instantiation of a communication system is developed. Consequently, a complete musical work can be conceptualized as a triadic sequence comprised of a creatively fabricated programatic component, a physically propagated absolute component, and a consciously perceived cognitive re-fabrication of the transmitted absolute component that provides a subjectively unique programatic component for each receiver or listener. These metaphorical descriptors ("programatic" and "absolute") are respectively chosen because cognitively a sound may be associated with, for example, any one or more colors, tastes, or emotions, whereas, in the external physical world sound is always an absolute consequence of only one type 
of event, atmospheric vibration.

In the absolute domain of the communication channel, it is hypothesised that an auditory signal can be partitioned by two quantitative measures. One is the periodicity and the other the variability of the acoustic waveforms. These may respectively be related to the 'semantic' and the esthetic properties of a given signal, with the type of source and the path taken by an acoustic signal determining the relative contribution made by each of these two measures. Thus, the musicality of an acoustic signal may be determined by the emergence of the organizational periodic or 'semantic' component from the more anarchistic esthetic or stochastic component (see [32]).

It is clear from this view, that the absolute and programatic components must be appropriately independent and sequentially arranged for a complete musical work to exist. Within the context of the reported framework, the digital record of the acoustic signal of several musical works representing different genres was analyzed by calculation of a redundancy measure of Shannon communication theory and the statistically based autocorrelation coefficient.

Results suggest that, with the exception of an acoustic signal formed by a single sine wave, it is the quantification of autocorrelation or periodicity rather than the redundancy or variability of an acoustic signal that better distinguishes between musical and nonmusical sound. It is predicted that an acoustic signal will likely start to loose musical character as the autocorrelation coefficient falls towards about $10 \%$ signal correlation or periodicity. This result suggests that in the absolute domain the organizing effect of the periodicity of an acoustic signal may be more important than its variability when a human listener is determining whether to interpret a signal as musical sound rather than noise.

As noted above, the meaning of a received acoustic signal is embedded in its cognitively located semantic and esthetic components. A tick signal indicating radioactive particle decay such as that provided by a Geiger counter is typically stochastic and usually distributed as a Poisson random variable. Within the framework presented here, such a signal would in the normal course of events be predicted to be classified as an esthetic phenomenon as it refers to a repertoire of knowledge associated with the given instrument. However, as the sound source is (cognitively) known to be from a particular instrument, the acoustic signal of the ticks becomes predominantly determined by its semantic associations rather than its esthetic nature. Thus, before reaching its cognitive destination, the stoachastic signal is ascribed a meaning that becomes intuitively translatable into action-the greater the frequency of ticks, the more dangerous the location. In this circumstance there is likely little practical reason for cognitively reverting attention to the stochastic esthetic component of the signal. As an audibly sonified stochastic signal, it embodies little musicality, but once the correct association is learned it can be cognitively elaborated into environmentally sourced meaning. This is in concordance with a previous observation that aesthetic investigation of the programatic domain must, above all, consider the beautiful object, and not the perceiving subject [33].

Furthermore, it is notable that in the normal course of events, a sound file containing a digitized acoustic signal of sufficient quality contains much of the information necessary to reconceptualize the essential elements of the original auditory environment from which the sound was obtained. Much of this environmental information, including aspects of force, rate, and material, etc, is represented in a digital sound recording. The point being that humans can refabricate significant aspects of the original acoustic environment from high quality digital recordings on the basis of innate cortical scene-analyzing functionality [34]. When represented as a time sequence of amplitudes, such complex three dimensional acoustic data becomes directly amenable to quantification by standard analytic methods and measures such as those proposed here.

Importantly, it is hypothesised that for sonification techniques to successfully create transparent and humanly interpretable acoustic signals that are not mistaken for noise, these signals should exhibit a minimal periodicity. Further, when some such lower bound is exceeded, suitable acoustic elaboration of a sound may provide a digital signal sufficient to support musification. This is not to suggest that the current aim is to provide a method that will prove one sonification is more effective than another.

A principled approach to the development of a musical work has also been presented. It implicitly provides a computationally based metaphor and framework for modelling human creative compositional processes. The approach originates in the recognition of what might be thought of as "objective historical markers," the penumbra of opinion surrounding the ideas of the absolute and the programmatic in music. In particular, here an attempt has been made to reconcile these two approaches to musical theory. On the one hand, there is the nature of music, its place in the cosmos, its physiological and psychological effects, and its proper use and cultural value; whereas on the other hand, there is the systematic description of materials and patterns of musical composition.

As expressed in the historical ideas of the absolute and programatic interpretations of musical works, a proposal has been made for a sonification methodology that satisfies various suggested requirements. The methodology outlined here appears appropriate for this end. It illuminates and enables the integrated development of what might be referred to as a general sonification methodology. It is an approach that provides a principled precursor for data musification and, putatively, the creation of what might be referred to as absolute program music. Importantly, the obverse is not being suggested, i.e. that musicality is necessary for effective sonification. It is rather that, as mentioned in the Introduction, effective sonification should in the first instance at least satisfy the requirement that it employs data as input and generates sound signals (but see the four associated caveats [10]).

To continue along this line, keeping accepted definitions of sonification in mind-at a technical level converting data to sound to gain knowledge, while at a perceptual level developing listening techniques for scientific enquiry-the approach endorsed here provides at least a principled methodology for further exploration of cognitive function, and elaboration of design principles and purpose, within a formal context or framework.

By initiating such a project at the level of basic nuances of human auditory perception, it is hypothesised that the path to several goals may be clarified. In particular, a more sophisticated understanding of (1) how human perception constrains sonification design, and (2) identification of the consequent guidelines for the creation of effective musification.

Finally, it is noted that it is only since the introduction and widespread availability of sophisticated digital technology and tools that a meaningful approach can be made towards more generalized models of the creative development of musical works through sonification and musification. It is hoped aspects of what is presented here will provide a preliminary contribution towards that goal. 


\section{REFERENCES}

[1] C. E. Shannon, The mathematical theory of communication, in The Mathematical Theory Of Communication, C. E. Shannon and W. Weaver, University of Illinois Press, Urbana \& Chicago, IL, U. S. A., 1963. pp. 31-125.

[2] C. Toumazou, J. B. Hughes, and N. C. Battersby, "Introduction," in C. Toumazou, J. B. Hughes, \& N. C. Battersby (Eds.), Switched-Currents: An Analogue Technique For Digital Technology, Peter Peregrinus Ltd., London, U. K., 1993, pp. 1-7.

[3] T. Hermann, A. Hunt, J. G Neuhoff (Eds.). "The Sonification Handbook," Logos Verlag, Berlin, Germany, 2011.

[4] G. Kramer, B. Walker, T. Bonebright, P. Cook, J. Flowers, N. Miner, and J. Neuhoff, "Sonification report: Status of the field and research agenda," Tech. Rep., Int. Community for Auditory Display, http://digitalcommons.unl.edu/cgi/viewcontent.cgi?article=14 43\&context=psychfacpub (accessed 29 Feburary 2016), 1997.

[5] S. Gresham-Lancaster, "Relationships of sonification to music and sound art," AI \& Soc., vol. 27, pp. 207-212, 2012.

[6] V. Straebel, "The sonification metaphor in instrumental music and sonification's romantic implications." in Proc. 16th Int. Conf. on Auditory Display, Washington, DC, U.S. A, June, 2010.

[7] J. Edlund, "The Virtues of the Musifier: A Matter of View," http://musifier.com/index.php/tech/musification-andview, (accessed 29 February 2016), 2004.

[8] S. Barrass, and G. Kramer, "Using sonification," Multimedia Syst., vol. 7, pp. 23-31., 1999.

[9] A. Schoon, "Sonification in music", in Proc. 15th Int. Conf. on Auditory Display, Copenhagen, Denmark, May , 2009.

[10] T. Hermann, "Taxonomy and definitions for sonification and auditory display," in Proc. 14th Int. Conf. on Auditory Display, Paris, France, June 2008.

[11] F. Visi, G. Dothel, D. Williams, and E. Miranda, "Unfolding Clusters: A Music and Visual Media Model of ALS Pathophysiology," in Proc. SoniHED Conf. Sonif. Health Env. Dat., York, U. K., 2014.

[12] A. Moles, Information Theory And Esthetic Perception, University of Illinois Press, Urbana, IL, U. S. A., 1966, p. 129.

[13] L. Goehr, The Imaginary Museum of Musical Works: An Essay In The Philosophy Of Music, Clarendon Press, Oxford, U. K., 2007.

[14] H. L. F. Helmholtz, Sensations Of Tone As A Physiological Basis For The Theory Of Music, A. J. Ellis (trans.), London, UK, 1912. p. 3.

[15] M. A. Arbib, ed., Language, Music, And The Brain: A Mysterious Relationship, The MIT Press, Cambridge, MA, U.S. A, 2013.

[16] J. Jeans, Science And Music, Cambridge University Press: London, UK, p. 83, 1961.

[17] A. Moles, Information Theory And Esthetic Perception, University of Illinois Press, Urbana, IL, U. S. A., 1966, p. 81.

[18] T. D. Rossing (ed.), Springer Handbook of Acoustics, Springer, New York, NY, U. S. A., 2007. pp. 747-748.
[19] E. Varèse, and W. Chou, "The liberation of sound," Perspectives of New Music, vol. 5, no. 1, pp. 11-19, p. 18, 1966.

[20] D. L. Hartmann, "ATM 552 Notes: Time Series Analysis - Section 6a," http://www.atmos.washington.edu/ dennis/552_Notes_6a.pdf, (accessed 29 February 2016), p. 128, 2016.

[21] Plato, The Republic, Book III, 398, in B. Jowatt (trans.), The Dialogues of Plato, Volume III, 3rd ed., The Clarendon Press, Oxford, U. K., 1892. p. 83.

[22] R. Hartley, Transmission of information. The Bell Syst. Tech. $J, 1928,7: 535-563$

[23] T. Xu, Chiu D and I. Gondra, "Constructing target concept in multiple instance learning using maximum partial entropy," in P. Perner (ed.), Machine Learning and Data Mining in Pattern Recognition, Springer, Berlin, Germany, 2012, pp. 169-182.

[24] J. W. Eaton, D. Bateman, and S. Hauberg, GNU Octave Version 3.8.0 Manual: A High-Level Interactive Language For Numerical Computations, CreateSpace Independent Publishing Platform.

[25] R. Cogan \& P. Escot, Sonic Design: The Nature of Sound and Music, Prentice Hall, Inc., Englewood Cliffs, NJ, U. S. A., 1976. p. 442.

[26] S. Gelfand, Hearing: An Introduction To Psychological And Physiological Acoustics, Marcel Dekker Inc, New York, NY, U.S. A., 1998. p. 281.

[27] J. E. Cohen, Information theory and music, Behav. Sci, 1962, vol. 7, no. 2, pp. 137-163.

[28] [25], p. 444.

[29] D. Crystal, Prosodic Systems And Intonation in English, Cambridge University Press, Cambridge, UK, 1969. p. 110.

[30] H. F. Olsen, Music, Physics, and Engineering, 2nd ed., Dover Publications, Inc, New York, NY, U. S. A., 1967. p. 383.

[31] Ovid, The story of Orpheus and Eurydicé, in Ovid's Metamorpheses, Book X. J. Dryden (trans.), Proprietors of the English Classics, London, UK, 1826. pp. 246-274. pp. 246-247.

[32] E. Wind, Art and Anarchy, 3rd ed., Gerald Duckworth \& Co. Ltd., London, U. K., 1985.

[33] E. Hanslick, The Beautiful In Music, G. Cohen (trans.), Novello and Company, London, U. K., 1891. p. 17.

[34] A. S. Bregman, Auditory Scene Analysis: The Perceptual Organization of Sound, A Bradford Book, The MIT Press, Cambridge, MA, U. S. A. 1990. p. 455. 